\title{
PET-saúde: gestão e atenção à saúde potencializando mudanças na formação
}

\author{
Maria Helena Rodrigues Galvão*, Cláudia Helena Soares De Morais Freitas**, Lucélia Lourenço

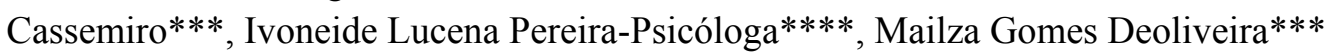 \\ * Estudante curso de Odontologia da Universidade Federal da Paraíba- \\ Bolsista PET \\ ** Professora de Saúde Coletiva do Departamento de Clínica e Odonto- \\ logia Social, Centro de Ciências da Saúde - Universidade Federal da \\ Paraíba - Tutora PET \\ *** Estudante do curso de Fisioterapia da Universidade Federal da Paraí- \\ ba - Bolsista PET \\ **** Gerente Operacional DST/AIDS/HEPATITES VIRAIS-SES-PB- \\ Preceptora PET \\ **** Assistente Social da Secretaria do Estado da Saúde da Paraíba - Pre- \\ ceptora PET
}

\section{RESUMO}

O Projeto PET-Saúde-Redes/ Eixo Vigilância em Saúde foi implantado na UFPB em parceria com a SES-PB, na perspectiva de superar lacunas e reorientar a formação dos profissionais da saúde. Este trabalho tem como objetivo relatar como a experiência no PET-SAÚDE potencializou o desenvolvimento das Competências Gerais propostas pelas DCN. As vivências no PET proporcionam a inserção dos estudantes em vários cenários de aprendizagem. $\mathrm{Na} \mathrm{Ge}-$ rência Operacional em DST/AIDS e Hepatites Virais foram vivenciadas as demandas estaduais de organização da rede de atenção, trabalhando as questões relacionadas à descentralização das ações; organização de fluxos dos usuários; recursos técnicos e humanos; educação permanente;e planejamento das ações, orientado pelo princípio da informação para a ação. Perante a atenção ambulatorial hospitalar, no trabalho com equipe multiprofissional, fez-se parte do processo de sensibilização dos profissionais em seu local de atuação. Logo,se propiciouo desenvolvimento da comunicação multiprofissional, liderança e, sobretudo, o contato com os usuários do serviço. No campo específico da odontologia foi possível obter conhecimento dos espaços de atuação extra clínica do cirurgião-dentista, em especial, na atenção ambulatorial e hospitalar voltada para a prevenção, promoção e reabilitação de saúde no contexto da AIDS e Hepatites Virais. O PET potencializa uma formação reorientada para as práticas de atenção, processos de trabalho, e a construção do conhecimento a partir das necessidades do serviço. Fortalecendo, portanto, a implantação das Diretrizes Curriculares Nacionais e a integração ensino-serviço.

Descritores: Educação em Odontologia; Prática Profissional; Recursos Humanos em Saúde.

\section{INTRODUÇÃO}

O processo de formação em saúde é um desafio atual, uma vez que a implantação recente e de caráter substitutivo de um 
novo Sistema de Saúde, o Sistema Único de Saúde (SUS), trouxe novos paradigmas para a atenção à saúde; fazendo-se evidente a necessidade de uma reorientação na formação profissional voltada para a atuação no SUS. O modelo de saúde hegemônico, o biomédico, caracterizado por privilegiar as ações curativas, em detrimento das preventivas, assim como, a fragmentação do conhecimento e a redução do indivíduo em partes, mostrou-se insuficiente para atender as necessidades da população.

Em razão disto, algumas estratégias que visam reduzir o descompasso entre o ensino superior, o SUS e a as necessidades de saúde da população estão sendo implementadas ${ }^{1}$. Dentre elas se sobressaem a Lei de Diretrizes e Bases da Educação de 1996, e posteriormente, as Diretrizes Curriculares Nacionais (DCN) para os cursos de graduação em saúde - aprovadas entre os anos de 2001 e $2004^{2,3,4,5,6}$.

Dentre outros elementos, as DCN estabelecem um novo perfil profissional, voltado para a produção do cuidado integral e com aptidão para trabalhar no SUS, haja vista que o formando/egresso deve possuir formação generalista, humanista, crítica e reflexiva; capaz de atuar em todos os níveis de atenção; afinado com a realidade social, com o processo de saúde-doença e comprometido com o ser humano. Além disso, os profissionais devem desenvolver habilidades e competências gerais que ultrapassam os limites do conhecimento técnico, algumas delas são: comunicação, liderança, atuação de maneira colaborativa/em equipe, tomada de decisões, educação permanen$\mathrm{te}^{3,4,5,6}$. E, ao estabelecerem as competências e habilidades gerais e específicas, enfatizam a formação em atitudes voltadas para a saúde, cidadania, comunidade e a atuação em equipe. Avançam, também, ao consolidarem, na estrutura do curso de graduação, a integração ensino-serviço, vinculando a formação profissional acadêmica às necessidades sociais da saúde, com ênfase no SUS $^{7}$.

Énesse contexto que surgeminiciativas governamentais,fruto da ação intersetorial dos Ministérios da Saúde e Educação, com pretensões de potencializar a reconfiguração da formação dos trabalhadores de saúde, assim como aperfeiçoar os recursos humanos vinculados ao SUS, por meio da educação permanente. São exemplos delas o Programa de Incentivo às Mudanças Curriculares nos Cursos de Medicina (Promed), o Programa Nacional de Reorientação da Formação Profissional em Saúde (PRÓSAÚDE) e o Programa de Educação pelo Trabalho para a Saúde (PET - Saúde).

O PRÓ- SAÚDE teve sua primeira edição no ano de 2005, incluindo os cursos de enfermagem, medicina e odontologia; sendo a segunda edição lançada em 2007 para os demais cursos. Esse programa busca promover a integração ensino-serviço, visando à reorientação da formação profissional, assegurando uma abordagem integral do processo saúde-doença com ênfase na atenção básica ${ }^{8}$.O Programa de Educação pelo Trabalho para a Saúde (PET - SAÚDE), criado em 2008, atua através do fomento à formação de grupos de aprendizagem tutorial em áreas estratégicas para o SUS, caracterizando-se como instrumento para a qualificação em serviço dos profissionais da saúde, bem como da iniciação ao trabalho e vivências dirigidas aos estudantes das graduações em saúde ${ }^{9}$.

O PET- Redes de Atenção em atual vigência (2012-2014) na Universidade Federal da Paraíba (UFPB) foi implantado com o objetivo de estimular mudanças curriculares com inserção dos estudantes nas Redes de Atenção à Saúde do SUS; desenvolver processos de formação e educação 
permanente com os profissionais dos diversos serviços da Rede; estimular e fortalecer iniciativas de mudança do processo de trabalho em saúde no caminho da integralidade; desenvolver pesquisas com base nas necessidades locais.

Dentre outros inseridos na instituição, encontra-se o projeto implantado em parceria com a Secretaria Estadual de Saúde da Paraíba (SES-PB) no ano de 2012, intitulado "A estruturação da rede de serviços para vigilância das doenças transmissíveis: DST/AIDS e Hepatites Virais". Este possui 1 tutor, 6 preceptores e 12 estudantes de 7 cursos de graduação em Saúde (odontologia, medicina, enfermagem, fisioterapia, nutrição, farmácia e terapia ocupacional). Entre os objetivos específicos desse projeto consistem em contribuir para a qualificação das informações sobre DST/AIDS- Hepatites virais no Estado da Paraíba; melhorar as notificações de DST/AIDS, Hepatites virais; subsidiar o planejamento, monitoramento e avaliação das ações; estabelecer fluxos de atendimento, de referência e contra referênciapara as DST/AIDS e Hepatites virais no Estado da Paraíba.

Este trabalho tem a finalidade de relatar como a experiência no PET-SAÚDE potencializou o desenvolvimento das Competências Gerais propostas pelas Diretrizes Curriculares Nacionais (DCN) do curso de Odontologia, bem como a contribuição dessas vivências no processo de formação profissional.

\section{METODOLOGIA}

As atividades efetuadas através do projeto PET- Saúde- Redes/ Eixo Vigilância em Saúde dispõem de vários cenários de prática, localizados na sede da Secretaria Estadual de Saúde da Paraíba (SES-PB) e no Complexo Hospitalar De Doenças Infecto Contagiosas Dr. Clementino Fraga
(CHCF), sendo este referência estadual no atendimento de portadores de AIDS, Hepatites Virais e agravos associados.

A organização operacional do grupo tutorial se deu através da formação de 6 grupos, sob a supervisão da tutora, contendo 2 estudantes e um preceptor. Cada grupo exerce atuação em um setor específico dos cenários de prática, à saber: Gerência Operacional em DST/AIDS e Hepatites Virais na SES-PB; e no CHCF os setores de Vigilância Epidemiológica Hospitalar, Fisioterapia, Ambulatório de Pneumologia e Assistência Domiciliar Terapêutica e Paliativa; havendo rodízio trimestral entre os estudantes.

O planejamento das atividades ocorreu em conjunto através de reuniões semanais com todos integrantes do grupo tutorial, mediante o estabelecimento de prioridades baseadas nas necessidades identificadas por estudantes e preceptores nos campos de atuação. Foi utilizada a pedagogia de problematização, com discussões fundamentadas em artigos científicos, leitura de documentos públicos e as vivências dos participantes; para o planejamento das ações específicas em cada campo de atuação e ações conjuntas envolvendo todo o grupo tutorial, estabelecendo metas e promovendo a avaliação dos resultados.

Além das atividades realizadas nos cenários de prática, foram desenvolvidas atividades extra muros de prevenção e educação,para a população geral acerca dos agravos em questão e campanhas de testagem e aconselhamento para AIDS, Hepatites B e C e Sífilis.

Foram também, executadas pesquisas científicas com o objetivo de qualificar as informações sobre DST/AIDS, Hepatites virais no Estado da Paraíba, utilizando os bancos de dados públicos e institucionais (SINAN, SICLOM, SIM, SISCEL) como 
ferramenta para o aprimoramento da prática profissional e melhoria dos processos de trabalho dos profissionais inseridos no serviço. Refletindo, deste modo, em uma melhor organização dos recursos disponíveis e ações a serem programadas; subsidiando assim o planejamento, monitoramento e avaliação das ações no Estado.

\section{RESULTADOS}

As ações desenvolvidas no Eixo Vigilância em Saúde do PET-Saúde-Redes incluem duas vertentes características dos cenários de prática, a gestão e a atenção em saúde, na perspectiva de redes.Tais ações são caracterizadas por promover uma ponte ideológica entre o serviço de atenção hospitalar, representado pelo $\mathrm{CHCF}$ e a gestão em DST/AIDS e Hepatites Virais. Criando, portanto, um espaço de comunicação de potencialidades e fragilidades, onde há a possibilidade de se discutir em equipe, estratégias para o aprimoramento da política de DST/AIDS no Estado.

A vivência na Gerência Operacional em DST/AIDS e Hepatites Virais SES/PB proporcionou a participação na elaboração da política de AIDS na perspectiva de construção da rede de atenção no Estado, destacando-se o processo de implantação de testes rápidos para AIDS, Sífilis, Hepatites B e $\mathrm{C}$ na atenção primária, nas Unidades de Saúde da Família de todo o Estado;com a qualificação de profissionais em todas as regionais de saúde da Paraíba. Outro aspecto da política de AIDS implementado foi a descentralização do fluxo de atendimento de portadores de AIDSpara os Serviços de Atendimento Especializados implantados em cidades satélites, antes concentradona capital do Estado, e a implementação do monitoramento dos Serviços de Atendimento Especializados atravésde bancos de dados considerando a notificação de agravos, mortalidade e dispensação de medicamentos antirretrovirais.

No decorrer de todas as atividades reconhecemos a importância de informações epidemiológicas fidedignas para o planejamento e efetividade das ações de saúde como a alocação de recursos técnicos e humanos, subsidiando a avaliação dos resultados obtidos. Para isso, faz-se uso do monitoramento das informações presentes nos bancos de dados, a fim de evitar duplicidades e subnotificações de casos de AIDS.

A atuação no $\mathrm{CHCF}$ permitiu uma articulação das ações, antes iniciadas sob outra esfera de governabilidade, especialmente na sensibilização dos profissionais em seu local de atuação através da educação permanente, tornando o PET-Saúde um agente de mudanças na organização do serviço de atenção. Em discussão com os técnicos do Núcleo de Vigilância Epidemiológica do hospital foram realizadas mudanças nos fluxos de informações para as notificações de agravos, sendo também possível intensificar a comunicação multiprofissional, destacando a importância da notificação, da informação e da confidencialidade; desta forma beneficiando o funcionamento geral do serviço tendo em vista a satisfação do usuário.Além destas, foram realizadas atividades de educação e prevenção em saúde com os usuários em atendimento, utilizando o tempo de espera, para a realização de palestras; com temas de prevenção ao câncer de mama e próstata, ressaltando a importância obtenção de hábitos saudáveis; dentre outras questões de interesse aos usuários e profissionais do serviço de saúde.

No âmbito da atenção à saúde, o desenvolvimento das ações de prevenção, promoção e prevenção da saúde, proporcionou o reconhecimento da importância do trabalho em equipe multiprofissional, não 
se restringindo às práticas específicas em saúde bucal, porém exercendo o trabalho de forma integrada e multiprofissional, formulando estratégias de enfrentamento a fim de solucionar questões inerentes à saúde tanto individual quanto coletiva.

Mesmo com o esforço que se vê em mudar o processo de ensino focado no profissional liberal para um profissional com perfil para o SUS, ainda há a fragilidade em se pensar que o cirurgião dentista é apenas um clínico, limitado a atuar em um consultório, seja ele público ou privado. Há uma lacuna na formação do cirurgião-dentista para a atenção hospitalar, e isto se reflete na prática, pois não desempenham as ações necessárias preconizadas pelaPolítica Nacional de Saúde Bucal e Política Nacional de AIDS.

Os cenários de prática dispõem de um espaço propício para o desenvolvimento da competência da Comunicação. Desde o início tratamos de informações sobre usuários portadores de AIDS, uma doença envolvida por um estigma social, sendo imprescindível o uso da confidencialidade durante a manipulação dos dados e na interação com os demais profissionais.

Além disso, trabalhar com os Sistemas de Informação em Saúde, confere experiências sobre a importância da alimentação e retroalimentação das informações produzidas nos serviços de saúde, uma atividade muitas vezes entendida como desnecessária, entretanto essencial para análise de indicadores de saúde, e que contribui para a qualidade das ações da vigilância em saúde, como instrumento de gestão, subsidiando os gestores no processo de planejamento e tomada de decisão.

\section{DISCUSSÃO}

É possível considerar queasDiretrizes Curriculares Nacionais (DCN) repre- sentam a ação institucional mais expressiva e abrangente a respeito da proposição de mudanças no cenário da formação profissional em saúde, e para isso toma por base o estabelecimento de competências gerais e específicas ${ }^{10}$. Podendo ser conceituadas como a capacidade de agir eficazmente em um determinado tipo de situação apoiada em seus conhecimentos, mas sem se limitar a eles ${ }^{11,12}$. Para tanto, faz-se uso da pedagogia das competências como principal fundamento pedagógico no texto das DCN.

Tal teoria pedagógica, afirma que o professor em particular e a escola como um todo não devem ofuscar o estudante; ao contrário, devem reforçar a capacidade do aluno construir o seu próprio aprendizado e, dessa forma, qualificar-se para escolher como se adaptar e como cooperar com o sistema, envolvendo, portanto o desenvolvimento de conhecimentos, atitudes e habilidades ${ }^{10,12,13,14,15}$.

As DCN do Curso de Graduação em Odontologia em seu artigo $4^{\circ}$ estabelecem as seguintes competências e habilidades gerais que devem ser preconizadas na formação profissional do Cirurgião Dentista, são elas: Atenção à saúde; Tomada de decisões; Comunicação; Liderança; Administração e gerenciamento; Educação permanente ${ }^{6}$.

A competência Tomada de Decisões estabelece que o trabalho dos profissionais de saúde deve estar fundamentado na capacidade de ponderar o uso das ferramentas disponíveis necessárias para a efetivação do seu trabalho, tomando as condutas adequadas baseadas em evidências científicas. Além desta, a competência de Administração e Gerenciamento, institui que os profissionais de saúde devem ter o ímpeto de tomar iniciativas e realizar a administração e o gerenciamento de todos os recursos envolvidos no seu processo de trabalho sejam 
eles físicos, materiais ou informações. Destaca-se também a competência de Liderança, que afirma que os profissionais de saúde devem estar aptos a assumir posições de liderança nas equipes de trabalho ${ }^{6}$. O cumprimento de tais competências garante a formação de profissionais preparados e flexíveis para atuar em meio à dinâmica dos serviços públicos de saúde.

As diretrizes de Tomada de Decisões, Liderança e Administração e gerenciamento permearam todo o processo de atuação. $\mathrm{O}$ fato de ter a oportunidade de ponderar custo-efetividade dos recursos existentes se fazendo presente no processo real de organização das redes de atenção em DST/AIDS e Hepatites Virais no Estado despertou para um campo de atuação promissor, o de gestão em serviços de saúde, e que não é comum o interesse dos graduandos, pois, há lacunas na formação de cirurgiões dentistas para atuação como gestores, empreendedores e transformadores da realidade.

De acordo com a competência de Atenção á Saúde os profissionais devem desenvolver de forma integrada ações de prevenção, promoção, proteção e reabilitação em saúde, nos níveis individual e coletivo, realizando seus serviços dentro dos mais altos padrões de qualidade. Já a competência da Educação Permanente estipula que, os profissionais devem ser capazes de aprender continuamente tanto na sua formação, quanto na sua prática; tendo responsabilidade com a sua educação e com o treinamento das futuras gerações de profissionais $^{6}$. Tais conceitos, se abordados durante a graduação contribuem para a formação de um profissional qualificado e capacitado para lidar com as atualizações do serviço, garantindo desta maneira um serviço de saúde de qualidade.
Referente à atenção odontológica hospitalar, componente da atenção integral á saúde, foi aprovado o Projeto de Lei $\mathrm{n}^{\circ}$ 2.776/08 que estabelece a obrigatoriedade da presença dos profissionais de odontologia nas Unidades de Terapia Intensiva. No entanto tal norma ainda não se encontra em vigor, pois não foi sancionada como $1 \mathrm{ei}^{16}$. Enquanto isso a Portaria $\mathrm{n}^{\circ} 706$, de 20 de julho de 2012 do Ministério da Saúde (MS), não estabelece o cirurgião dentista como integrante da equipe mínima nos serviços especializado das redes de atenção á saúde, com exceção do serviço de Pronto Atendimento Odontológico, sendo este profissional excluído inclusive da equipe mínima necessária a um Pronto Socorro Odontológico $^{17}$. Da mesma forma a portaria $\mathrm{n}^{\circ}$ 2.809, de 7 de dezembro de 2012 do MS, não inclui o cirurgião dentista na equipe multidisciplinar nas Unidades de Internação em Cuidados Prolongados (UCP) dentro de um Hospital Geral ou Especializado ou Hospital Especializado em Cuidados Prolongados (HCP), embora afirme que para a habilitação de tais serviços faz-se necessária a oferta de atendimento odontológico ${ }^{18}$. Entretanto, o MS garante recursos para a atenção hospitalar odontológica, resguardados pela Portaria $\mathrm{n}^{\circ} 1.032$ de 05 de maio de 2010 do MS, apenas para o atendimento de pacientes com necessidades especiais ${ }^{19}$.

A Educação Permanente, entretanto, possui uma definição que vai além da competência prevista nas DCN. Como uma política específica do Ministério da Saúde, a Política Nacional de Educação Permanente em Saúde, constitui como uma estratégia do SUS para a formação e o desenvolvimento de trabalhadores para o setor da saúde. Considerada como um conceito pedagógico, no setor da saúde, efetua relações orgânicas entre ensino e ação nos serviços e 
entre docência e atenção à saúde, para as relações entre formação e gestão setorial, desenvolvimento institucional e controle social em saúde. Realizando, portanto, a agregação entre aprendizado e reflexão crítica sobre o trabalho, resolutividade da clínica e promoção da saúde coletiva ${ }^{20,21}$.

\section{CONSIDERAÇÕES FINAIS}

Embora tenham se passado 25 anos de sua homologação, o SUS, ainda enfrenta desafios quanto àimplementação de seus princípios e diretrizes. E muitos destes perpassam pelo processo de formação dos profissionais de saúde. O PET potencializa uma formação reorientada para as práticas de atenção preconizadas pelo SUS, o processo de trabalho interdisciplinar, e a construção do conhecimento a partir das necessidades do serviço; fortalecendo a implantação das Diretrizes Curriculares Nacionais e a integração ensino-serviço como ferramenta de mudança de paradigma no processo de formação dos profissionais em saúde.

Acreditamos o que um dos maiores impactos do programa PET-Saúde Redes foi a possibilidade de desenvolvimento do trabalho de ensino e pesquisa de intervenção construído e executado conjuntamente entre as instituições de ensino e serviço, fortalecendo o compromisso das instituições para a formação de um profissional que venha atender as necessidades de saúde da população.

Além disso, no convívio com os estudantes, os profissionais da rede de serviços são valorizados pela oportunidade de acompanhar os estudantes no trabalho, inseridos numa equipe de saúde, com possibilidade de formação eestímulo ao estudo, à atualização, à transformação. As pesquisas proporcionam aos profissionais ferramentas para melhorar seu trabalho na gestão, no atendimento ao usuário e também promovem o envolvimento da equipe.

É importante destacar que apesar da falta de governabilidade do PET-SAÚDE para lidar com a resolução de vários problemas identificados, estes foram discutidos com a gestão e encaminhados.

As ações do PET-SAÚDE estão diretamente imbricadas com a Educação Permanente, onde os profissionais devem se comprometer em acompanhar as evoluções dos processos de trabalho, incorporando novas tecnologias e comprometendo-se com a sua educação e com a educação dos futuros profissionais, entendendo o conhecimento construído no exercer da sua profissão como bem que será mais útil se compartilhado.

O PET potencializa uma formação reorientada para as práticas de atenção, processos de trabalho, e a construção do conhecimento a partir das necessidades do serviço;possibilitando em todo tempo a troca de saberes, a reflexão das atividades no serviço, o repensar das práticas e a superação das dificuldades no processo de trabalho. Transformando, por fim, o proceder, as atitudes e o trabalho em equipe,fortalecendo, portanto, a implantação das Diretrizes Curriculares Nacionais e a integração ensino-serviço.

\section{REFERÊNCIAS}

1. González AD, Almeida MJ. Movimentos de mudança na formação em saúde: da medicina comunitária às diretrizes curriculares. PhysisRevista de Saúde Coletiva, Rio de Janeiro, 20 [ 2 ]: 551570, 2010.

2. Brasil. Lei n. 9.394, de 20 de dezembro de 1996. Estabelece as diretrizes e bases da educação nacional. Diário Ofici- 
al da União, Brasília, DF, 23 dez.1996. Seção1, p.27.833.

3. Resolução CNE/CES 4/2001, de 7 de novembro de 2001a. Institui Diretrizes Curriculares Nacionais do Curso de Graduação em Medicina. Disponível em: http://portal.mec.gov.br/cne/arquivos/p df/CES04.pdf. Acesso em: 11 de setembro de 2012.

4. Resolução CNE/CES 3/2001, de 7 de novembro de 2001b. Institui Diretrizes Curriculares Nacionais do Curso de Graduação em Enfermagem. Disponível

em: http://portal.mec.gov.br/cne/arquivos/p df/CES03.pdf. Acesso em: 11 de setembro de 2012.

5. Resolução CNE/CES 4/2002, de 19 de fevereiro de 2002a. Institui Diretrizes Curriculares Nacionais do Curso de Graduação em Fisioterapia. Disponível em: http://portal.mec.gov.br/cne/arquivos/p df/CES042002.pdf. Acesso em: $11 \mathrm{de}$ setembro de 2012.

6. Resolução CNE/CES 3/2002, de 19 de fevereiro de 2002b. Institui Diretrizes Curriculares Nacionais do Curso de Graduação em Odontologia. Disponível em: Acesso em: http://portal.mec.gov.br/cne/arquivos/p df/CES032002.pdf. 11 de setembro de 2012.

7. Lampert JB. Avaliação institucional nos cursos de graduação da área de saúde: Avaliar o quê e para quê? Cadernos ABEM, Rio de Janeiro, 5: 42-55, 2009.

8. Brasil. Ministério da Saúde. Ministério da Educação. Programa Nacional de Reorientação da Formação Profissional em Saúde - Pró-Saúde: objetivos, implementação e desenvolvimento poten- cial / Ministério da Saúde, Ministério da Educação. - Brasília: Ministério da Saúde, 2007.

9. Brasil. Portal da Saúde/PET-Saúde. Disponível em: http://portal.saude.gov.br/portal/saude/ profissional/visualizar texto.cfm?idtxt=35306. Acesso em: 03 de outubro de 2013.

10. Pereira IDF, Lages I. Diretrizes curriculares para a formação de profíssionais de saúde: competências ou práxis? Trabalho, Educação e Saúde, Rio de Janeiro, 11 [ 2 ]: 319-338, 2013.

11. Carvalho ACP. Planejamento do curso de graduação de Odontologia. Revista ABENO, Rio de Janeiro, 4 [ 1 ]: 7-13, 2004.

12. Perrenoud P. Construir as competências desde a escola. PortoAlegre: Artmed; 1999. $90 \mathrm{p}$.

13. Ghiraldelli JRP. Richard Rorty: a filosofia do novo mundo em busca de mundos novos, Petrópolis: Vozes; 1999.

14. Ramos MN. A pedagogia das competências: autonomiaou adaptação? São Paulo: Cortez, 2006.

15. _. É possível uma pedagogia das competências contra-hegemônica? Relações entre pedagogia das competências, construtivismo e neopragmatismo. Trabalho, Educação e Saúde, Rio de Janeiro, 1 [ 1 ]: 93-114, 2003.

16. Brasil. Projeto de Lei $n^{\circ} 2776 / 2008$. Disponível em: http://www.camara.gov.br/proposicoes Web/fichadetramitacao?idProposicao= $\underline{383113}$. Acesso em: 10 de janeiro de 2014.

17. . Ministério da Saúde. Portaria $\mathrm{n}^{\mathrm{o}}$ 706, de 20 de julho de 2012. Disponível

em:http://bvsms.saude.gov.br/bvs/saud 
ele-

gis/sas/2012/prt0706 $20 \quad 07 \quad 2012 . h t m l$

. Acesso em: 10 de janeiro de 2014.

18. _. Ministério da Saúde. Portaria $\mathrm{n}^{\mathrm{o}}$

2.809 , de 7 de dezembro de 2012. Disponível

em:http://bvsms.saude.gov.br/bvs/saud

ele-

gis/gm/2012/prt2809 $07 \quad 12$ 2012.htm

1Acesso em: 10 de janeiro de 2014.

19. Ministério da Saúde. Portaria $n^{\circ}$

1.032, de 5 de maio de 2010. Disponível

em:http://bvsms.saude.gov.br/bvs/saud

ele-

gis/gm./2010/prt1032 $05 \quad 05 \quad 2010 . h t$

ml. Acesso em: 10 de janeiro de 2014.

20. Brasil. Ministério da Saúde. Portaria $N^{\circ}$ 198/GM, de 13 de fevereiro de 2004. Disponível

em: http://dtr2001.saude.gov.br/sas/PORTA RIAS/Port2004/GM/GM-198.htm

Acesso em: 23 de Janeiro de 2014.

21. Morita MC, Kriger L, Carvalho ACP, Haddad AE. Implantação das Diretrizes Curriculares Nacionais em Odontologia. Maringá: Dental Press Editora, 2007.

\section{ABSTRACT \\ PET-health: management and health care changes in training potentiating}

The PET-Health-Network /Axis Health Surveillance Project was implemented in UFPB in partnership with SES-PB, the perspective of overcoming gaps and reorientthe training of health professionals.
This work aims to report as experience in PET-HEALTH enhanced the development of the General Competencies proposed by DCN. The experiences in PET provide the integration of students in several learning scenarios. In operational Management in STD /AIDS and Viral Hepatitis experienced the state demands of organizing of care network, working the issues related to decentralization of actions, organizing the flows of users, technical and human resources, continuing education, action planning, oriented the principle of information for action. In hospital ambulatory care in working with multidisciplinary team, became part in the sensitization process of the professionals in the locale, being conducive to the development of multidisciplinary communication, leadership, and especially incontactwithservice users. In the specific field of dentistry it was possible meet spaces of acting extra clinic of the dentalsurgeon and their performance in ambulatory and hospital care focused on prevention, health promotion and rehabilitation in the context of AIDS and Viral Hepatitis. The PET enhances the training refocused on care practices, the work process, and the construction of knowledge based service needs, strengthening the implementation of the National Curriculum Guidelines and the integration teaching-service.

Descriptors: Education Dental; Professional Practice; Health Manpower. 